\title{
Mammography: EUSOBI recommendations for women's information
}

\author{
Francesco Sardanelli • Thomas H. Helbich • \\ for the European Society of Breast Imaging (EUSOBI)
}

Received: 22 April 2011 /Revised: 20 August 2011 /Accepted: 9 September 2011 /Published online: 28 October 2011

(C) European Society of Radiology 2011

\begin{abstract}
This paper summarises the basic information to be offered to women who undergo mammography. After a delineation of the general aim of early diagnosis of breast cancer, the main difference between screening mammography and diagnostic mammography is explained. The best time for scheduling mammography in fertile women is defined. The need to bring images and reports from the previous mammogram (and from other recent breast imaging examinations) is highlighted. The technique and procedure of mammography are briefly described with particular attention to discomfort and pain experienced by a fraction of women who undergo the test. Information is given on the recall during a screening program and on the request for further work-up after a diagnostic mammography. The logic of the diagnostic mammography report and of classification systems such as BI-RADS and R1-R5 is illustrated, and brief but clear information is given about the diagnostic performance of the test, with particular reference to interval cancers. Moreover, the breast cancer risk due to radiation exposure from mammography is compared to the reduction in mortality obtained with the test, and the concept of overdiagnosis is presented. Finally, five frequently asked questions are answered.
\end{abstract}

\footnotetext{
F. Sardanelli $(\bowtie)$

Dipartimento di Scienze Medico-Chirurgiche, Università degli Studi di Milano, Unità di Radiologia, IRCCS Policlinico San Donato, Piazza Edmondo Malan 2,

20097 San Donato Milanese, Italy

e-mail: francesco.sardanelli@unimi.it
}

T. H. Helbich

Department of Radiology, Division of Molecular and Gender Imaging, Medical University of Vienna,

Vienna, Austria

\section{Introduction}

Malignant tumours (cancers) and benign diseases are very common in the breast. Aside from clinical history (conversation about disorders in the family, previous breast diseases/surgery, hormone therapy, personal well-being and complaints), inspection (external viewing) and palpation, which compose the so-called clinical breast examination, imaging procedures and especially mammography are of crucial importance in the detection of breast cancer and diagnosis of breast diseases.

Mammography is radiography of the breast. Its purposes are (1) early detection of breast cancer before symptoms (screening mammography) and (2) diagnosis in patients with symptoms such as a palpable lump (diagnostic or clinical mammography). The general aim is to enable early treatment of breast cancer, to improve survival rates and to reduce the need for aggressive treatment such as mastectomy $[1,2]$.

\section{Screening mammography and diagnostic mammography}

Mammography is the most important imaging procedure for breast cancer detection and diagnosis. It can be performed in a screening setting or a diagnostic setting.

Screening mammography Screening is performed periodically in order to find small cancers before they are detected through self-palpation or clinical breast examination. It is performed every 1,2 , or 3 years (depending on age, the regional screening programme, or the advice of one's doctor), from the age of 40-50 years until around 70-75. Relevant differences in screening programmes across Euro- 
pean countries, including ways of reporting, are due to differences in culture, technical circumstances, biopsy options, financial restrictions and breast cancer prevalence. Women with a high frequency of breast cancer in their family should start even earlier with periodic imaging, possibly using magnetic resonance imaging [3], after consulting specialised centres, since mammograms in this age group have very limited value due to the higher density of breasts. Screening mammography is a standardised procedure composed of four views, two for each breast. It can be performed by a radiographer alone, and the resulting images are usually read by two radiologists, independently, in separate sessions. If the exam is judged negative (no suspicious findings), the woman receives a letter communicating this result. If something suspicious is found, the woman is recalled for a tailored further assessment (additional mammographic views, ultrasound and/or needle biopsy).

Diagnostic mammography This is performed in patients presenting with clinical symptoms such as a palpable lump, nipple discharge, or skin or nipple retraction, in order to diagnose or exclude breast cancer. It should be performed by a radiographer under the supervision of a radiologist. Before acquiring the four standard projections, a full clinical breast examination is performed, usually by the same radiologist, or the radiologist must have the results of a full clinical breast examination recently performed by another doctor. All palpable abnormalities and scars from previous surgeries are highlighted by attachment of a marker to the skin. If necessary, additional views are acquired after the standard procedure. A formal written report is prepared by the radiologist with conclusions, including recommended further steps.

Note A. If you have a planned screening mammography and you notice relevant symptoms, go to your radiologist in order to decide if you need a diagnostic mammography. However, if you have symptoms and you are getting a screening mammography, inform the radiographer about them! The radiologists reading your images will decide whether you should be recalled based on these symptoms. In any case, if your symptoms do not disappear, you should consult your radiologist even if your mammography has been judged negative.

\section{Scheduling/precautions}

The best time for mammography to be carried out is from day 7 to day 12 after the beginning of the woman's last menstruation. This is due to cyclical variations in breast density. No particular scheduling is required after meno- pause. If the woman is pregnant, ultrasound is preferred as first option.

Note B. You should bring images and reports from the previous mammogram (and from other recent breast imaging examinations) and give these to the radiographer or to the radiologist before the procedure. This can be crucial for image interpretation, due to the fact that some cancers are diagnosed only on the basis of changes that have occurred since a previous exam.

\section{Technique/procedure}

Mammography is performed using a dedicated X-ray unit. A particular radiographic technique is used which requires the compression of the breast for 5-10 $\mathrm{s}$ in order to deliver a low radiation dose and to obtain highquality images. It is standard practice to take two views per breast. Further additional views (projections) are acquired in special cases.

The procedure is performed with the woman's upper body undressed. All foreign objects (such as bras, necklaces, piercings, etc.) must be removed before the procedure. The woman will stand in an upright position in front of the machine. For each projection of each breast, the radiographer will place the breasts on the plate and will apply a progressive compression for 5-10 s. During breast squeezing, women may feel some pain or discomfort [4]. It is important not to move during this short time. Immediately after acquiring the mammogram, the breast will be released from compression. The entire bilateral standard procedure, including preparation, takes approximately 5-10 min.

Note C. To reduce pain or discomfort due to compression and to get the best mammograms, you should relax during the procedure; in particular, pectoral muscles should be relaxed. Follow the radiographer's instructions exactly, and bear in mind that heavier compression means a lower X-ray dose, higher image quality, and easier diagnosis. If you previously experienced a painful mammography in the premenstrual phase, try to arrange the next one from day 7 to day 12 of your cycle.

\section{After the procedure}

When the procedure is over, the woman returns to the waiting room. In the case of screening mammography, she is usually only informed whether or not the acquired images are technically adequate. If no views need to be repeated, she may leave. She will receive a letter communicating either that the mammogram is negative or that further assessment is needed (recall). 
The first event is far more probable (over $90-95 \%$ of cases). In the case of diagnostic mammography, after checking technical adequacy, the radiologist informs the patient either that the exam is completely negative or that further assessment is needed, which may include additional mammographic views, ultrasound, magnetic resonance imaging, and/or needle biopsy. In the diagnostic setting, the second event is more probable.

Note D. If you are recalled after a screening mammogram or you are asked to have an ultrasound after diagnostic mammography, this does not mean that you have cancer. The most probable result of this second examination is a higher level of certainty in stating that you do not have cancer! Less than 10\% of women who are recalled at screening are finally diagnosed with cancer. However, if a cancer is present, you would rightly like it to be diagnosed as early as possible.

\section{Mammography report and classification systems}

Diagnostic mammography and also diagnostic assessment of recalled women after mammography screening should be formally carried out by a certified breast radiologist. A detailed report should include a description of the clinical context, if relevant, as well as image findings, including breast density and structure according to different classification systems, interpretation and a final conclusion with recommendations.

In many European countries, standardised classification systems for the conclusions of mammography reports are used. A European system uses the five-level scale from R1 to R5 (where R stands for radiography). R1 means no abnormalities, R2 benign findings, R3 equivocal findings, R4 suspected cancer, R5 strongly suspected cancer. A system developed in the United States [Breast Imaging Reporting and Data System (BIRADS)] includes a similar scale from BIRADS 1 to BI-RADS 5, with the same meaning for scores 1, 2, 4, and 5. The main difference is in BI-RADS 3, which means a very low probability of cancer (less than $2 \%$ ), allowing the possibility of waiting for a short-interval (usually 3-6 months) before a repeat mammogram. The R3 category indicates a probability of cancer that is higher than BI-RADS 3. Moreover, the BI-RADS score system also includes BIRADS 0 (examination insufficient for a diagnostic conclusion; further work-up needed) and BI-RADS 6 (evaluation of an already diagnosed cancer).

Note E. In practice, if you have an R4-R5 or BI-RADS 4-5 finding, needle biopsy is recommended. In case of $R 3$ or BI-RADS 3, meet your radiologist and ask for a detailed explanation of this result, of the risks and of the probabilities associated with different options.

\section{Diagnostic performance of mammography}

No diagnostic test is perfect. This rule also applies to mammography. When thinking about screening, women should be aware that about one-third of cancers can be missed, especially in pre-menopausal women and in those with dense breasts or those with a lot of breast tissue who also have a higher risk for breast cancer [5, $6]$.

Note F. Do not underestimate the importance of breast symptoms (especially a new palpable lump, skin/nipple retraction or nipple discharge), regardless of the timing of your last negative screening mammography. Go to your radiologist and ask for a visit. Tell her/him your symptoms and she/he will decide the best course of action for you.

Conversely, not all suspicious findings visualised on a mammogram are cancers: depending on the level of suspicion, cancer is confirmed in $3-95 \%$ of the cases. When the suspicion is confirmed after further assessment, image-guided needle biopsy is mandatory before planning the treatment.

Note G. A suspicious mammographic finding is not a confirmed cancer. However, do not postpone further assessment and, if necessary, needle biopsy.

\section{Radiation exposure from mammography}

The radiation exposure for a mammogram is very low. A recent study [7] reported that undergoing annual mammography from age 40 to 55 years and biannual mammography from 56 to 74 years entails a risk of radiation-induced breast cancer equal to 1 in every 1,000 women screened. The risk of breast cancer in the female population is equal to at least one in every ten women. The first risk is 100 times smaller than the second while the reduction in breast cancer mortality thanks to early detection with screening mammography is equal to 44 $48 \%$ [8, 9]. In practice, the decision to undergo screening mammography halves the risk of death from breast cancer while increasing the risk of breast cancer by only $0.1 \%$.

\section{Overdiagnosis}

Not all the breast cancers diagnosed with screening are aggressive and fatal cancers. In the absence of screening mammography, some of them (probably 5-20\%) would have remained totally free of symptoms [10]. However, these cancers cannot be distinguished from those that, if 
left undiagnosed and untreated, would be fatal. Thus, if we want to reduce breast cancer mortality, we must accept a rate of overdiagnosed cancers with the consequence of a rate of unnecessary treatments.

\section{Five frequently asked questions (FAQs)}

How painful is breast compression?

Mammography is painless for about $40-50 \%$ of women, a little painful for $40 \%$, rather painful for $12 \%$, and very painful only for $4 \%$. Pain disappears immediately after the procedure for $76 \%$ of the women, while it lasts several minutes for $13 \%$, several hours for $7 \%$, and more than 1 day for $4 \%$ [4]. However, the advantages of compression are clear, and unnecessary pain may sometimes be avoided by suitable scheduling (see Note C).

When should the first mammogram be done? What time intervals for further examinations?

Different recommendations are issued by different radiological and cancer societies. There is a general agreement on the usefulness of screening mammography from 50 to 70 years of age, with a time interval depending on several factors described above. Extension from about 45 to about 75 is now adopted by several screening programmes [11]. When starting at 40, a 1-year interval can be recommended up to $45-50$, considering the probable higher density and the possible faster growth of the tumor. After 50, the optimal interval may be decided based on personal history and breast density.

Note H. If you are invited to attend an organised screening programme, follow the programme's planned interval. If you have any doubts about this time interval, or the usefulness of ultrasound as a supplemental screening method, consult your radiologist. If there has been a high number of incidences of breast cancer in your family, especially at a young age and before menopause, consult your radiologist and/or a specialised centre (e.g. a family cancer clinic).

What about screening mammography for women over 75 ?

The continuous increase in life expectancy prevents the definition of an upper age limit for screening mammography. A general suggestion is to continue screening with mammography for elderly women as long as their health is not significantly compromised by illness that drastically reduces life expectancy [11]. Discuss this decision with your radiologist.
Can women with breast implants or breast reconstruction undergo mammography?

Yes, in the majority of cases they can. Special views with back placement of the implant are commonly needed, as well as specific technical experience. Exceptions where mammography cannot be performed are breast reconstructions after complete removal of gland tissue. Mammography limitations due to the presence of implants can be counteracted by an accurate clinical breast examination and breast ultrasound.

Note I. Always tell the radiologist and/or the radiographer if you have one or two breast implants.

Is X-ray radiation from mammography dangerous?

The X-ray radiation associated with a mammogram is very low. See section "Radiation exposure from mammography" for a comparison between the risk and the reduction of breast cancer mortality due to mammography.

\section{References}

1. de Koning HJ (2003) Mammographic screening: evidence from randomized controlled trials. Ann Oncol 14:1185-1189

2. Feig SA (2005) Screening mammography controversies: resolved, partly resolved, and unresolved. Breast J 11:S3-S6

3. Sardanelli F, Boetes C, Borisch B et al (2010) Magnetic resonance imaging of the breast: recommendations from the EUSOMA working group. Eur J Cancer 46:1296-1316

4. Drossaert CH, Boer H, Seydel ER (2002) Monitoring women's experiences during three rounds of breast cancer screening: results from a longitudinal study. J Med Screen 9:168-175

5. Törnberg S, Kemetli L, Ascunce N et al (2010) A pooled analysis of interval cancer rates in six European countries. Eur J Cancer Prev 19:87-93

6. Corsetti V, Houssami N, Ghirardi M et al (2011) Evidence of the effect of adjunct ultrasound screening in women with mammography-negative dense breasts: interval breast cancers at 1 year follow-up. Eur J Cancer 47:1021-1026

7. Yaffe MJ, Mainprize JG (2011) Risk of radiation-induced breast cancer from mammographic screening. Radiology 258:98-105

8. Allgood PC, Warwick J, Warren RM et al (2008) A casecontrol study of the impact of the East Anglian breast screening programme on breast cancer mortality. Br J Cancer 98:206-209

9. Puliti D, Miccinesi G, Collina N, IMPACT Working Group, et al (2008) Effectiveness of service screening: a case-control study to assess breast cancer mortality reduction. Br J Cancer 99:423-427

10. Biesheuvel C, Barratt A, Howard K, Houssami N, Irwig L (2007) Effects of study methods and biases on estimates of invasive breast cancer overdetection with mammography screening: a systematic review. Lancet Oncol 8:1129-1138

11. Peek ME (2003) Screening mammography in the elderly: a review of the issues. J Am Med Womens Assoc 58:191-198 